\title{
Fatty acid-amino acid conjugates are essential for systemic activation of salicylic acid-induced protein kinase and accumulation of jasmonic acid in Nicotiana attenuata
}

\author{
Christian Hettenhausen ${ }^{1}$, Maria Heinrich ${ }^{2}$, lan T Baldwin ${ }^{2}$ and Jianqiang $\mathrm{Wu}^{{ }^{*}}$
}

\begin{abstract}
Background: Herbivory induces the activation of mitogen-activated protein kinases (MAPKs), the accumulation of jasmonates and defensive metabolites in damaged leaves and in distal undamaged leaves. Previous studies mainly focused on individual responses and a limited number of systemic leaves, and more research is needed for a better understanding of how different plant parts respond to herbivory. In the wild tobacco Nicotiana attenuata, FACs (fatty acid-amino acid conjugates) in Manduca sexta oral secretions (OS) are the major elicitors that induce herbivory-specific signaling but their role in systemic signaling is largely unknown.

Results: Here, we show that simulated herbivory (adding M. sexta OS to fresh wounds) dramatically increased SIPK (salicylic acid-induced protein kinase) activity and jasmonic acid (JA) levels in damaged leaves and in certain (but not all) undamaged systemic leaves, whereas wounding alone had no detectable systemic effects; importantly, FACs and wounding are both required for activating these systemic responses. In contrast to the activation of SIPK and elevation of JA in specific systemic leaves, increases in the activity of an important anti-herbivore defense, trypsin proteinase inhibitor (TPI), were observed in all systemic leaves after simulated herbivory, suggesting that systemic TPI induction does not require SIPK activation and JA increases. Leaf ablation experiments demonstrated that within 10 minutes after simulated herbivory, a signal (or signals) was produced and transported out of the treated leaves, and subsequently activated systemic responses.
\end{abstract}

Conclusions: Our results reveal that N. attenuata specifically recognizes herbivore-derived FACs in damaged leaves and rapidly send out a long-distance signal to phylotactically connected leaves to activate MAPK and JA signaling, and we propose that FACs that penetrated into wounds rapidly induce the production of another long-distance signal(s) which travels to all systemic leaves and activates TPI defense.

Keywords: Defense, Fatty acid-amino acid conjugates, Herbivore, Jasmonic acid, Mitogen-activated protein kinase (MAPK), Nicotiana attenuata, Systemic response

\section{Background}

Herbivores pose a major threat to plants. To cope with this challenge, plants have evolved sophisticated defense systems to perceive damage and herbivore-derived elicitors (the so-called herbivore-associated molecular patterns, HAMPs) [1] and activate a chain reaction of downstream

\footnotetext{
*Correspondence: wujianqiang@mail.kib.ac.cn

${ }^{1}$ Kunming Institute of Botany, Chinese Academy of Sciences, 650201

Kunming, China

Full list of author information is available at the end of the article
}

signaling events, including rapid activation of mitogenactivated protein kinases (MAPKs) [2-4], biosynthesis of phytohormones, such as jasmonic acid (JA), JA-isoleucine conjugate (JA-Ile), and ethylene [5], and reshaping transcriptomes, proteomes, and metabolomes.

It is believed that systemic responses prevent insects from escaping plant defense by moving to undefended tissues. Systemic defense was first discovered in tomato (Lycopersicon esculentum): after wounding, a signal was found to move to other parts of the plants and induce 
the production of an important defensive compound, proteinase inhibitor I (PI-I) [6]. In a wild tobacco, Nicotiana attenuata, in addition to PIs, transcriptional and metabolomic analyses indicated that various genes and metabolites are also up-regulated in systemic undamaged leaves and roots [7-9]. MAPKs and the phytohormones JA and JA-Ile are all upstream signaling molecules, which play important roles in regulating plant resistance to herbivores [3,4,10-13]. Wounding or herbivory activates MAPKs within a few minutes $[3,4,14,15]$ and rapidly induces the biosynthesis of JA, with levels peaking within 1-2 h $[16,17]$.

In tomato, cultivated tobacco, forage and turf grasses, rapid MAPK activation was also detected in certain systemic leaves after wounding [18-20]; however, wounding or treatment of simulated herbivory (wounding and application of herbivore oral secretions to wounds) did not result in changes of MAPK activity in the adjacent systemic leaf in $N$. attenuata [3], suggesting that systemic activation of MAPKs might be species-specific or dependent on leaf positions. Recently, it was found that wounding rapidly induces JA accumulation in systemic leaves in Arabidopsis [21,22]. In contrast, wound treatment did not induce the accumulation of systemic jasmonates in $N$. attenuata, but increased JA and JA-Ile levels were found in systemic leaves after simulated herbivore feeding $[23,24]$. Therefore, in addition to a long-distance signal that induces the accumulation of defensive compounds such as PIs in systemic leaves, another (or the same) signal or several signals rapidly travel to distal leaves and activates MAPK signaling and JA biosynthesis. A prerequisite for obtaining deeper insight into the molecular mechanisms underlying systemic defense is a thorough description of the spatial and temporal herbivory-induced responses in local and systemic leaves.

The wild tobacco, $N$. attenuata, is a diploid annual plant that inhabits the deserts of western North America. $N$. attenuata has been intensively studied in the aspect of how it responds to herbivory of the specialist insect Manduca sexta [25]. Feeding of M. sexta elicits the production of plant defense metabolites not only in the local leaves but also in systemic leaves distal to the wound sites [26]. Previous research on Arabidopsis, tomato, and tobacco has suggested that MAPK and JA signaling are involved in systemic responses [18,19,22-24]; however, most studies only focused on a rather limited number of systemic leaves and examined the responses either on the signaling or metabolite level. Here we comprehensively investigated the changes in MAPK activity, accumulation of JA/JA-Ile, as well as the levels of trypsin protease inhibitors (TPI), a typical systemic defense in Solanaceae, in local and systemic leaves after wounding and simulated herbivore treatments. We found that a rapid mobile signal induces salicylic acid-induced protein kinase (SIPK) activation and JA/JA-Ile accumulation in certain, but not all, systemic leaves in $N$. attenuata, and the production of this signal is highly dependent on fatty acid-amino acid conjugates (FACs) in M. sexta oral secretions (OS) that are introduced into wounds during feeding; furthermore, neither wounding nor FACs alone can induce elevated SIPK activity and JA/JA-Ile levels in systemic leaves. Using TPI activity assay and leaf ablation approach, we demonstrate that the pattern of TPI induction is different from that of systemically induced SIPK and JA/JA-Ile, and we propose that another signal travels at a similar speed to almost all systemic leaves to activate TPI biosynthesis.

\section{Results}

Simulated $M$. sexta herbivory treatment induces a specific spatial and temporal pattern of JA accumulation in Nicotiana attenuata systemic leaves

Given the central role of JA in regulating plant resistance to herbivores, we first examined whether simulated herbivore feeding induces systemic JA production. Because JA-Ile, the conjugate of JA and isoleucine, but not JA itself, functions as the active jasmonate hormone [27], the concentrations of JA-Ile were also determined. Slightly elongated plants (about $10 \mathrm{~cm}$ in height, Figure 1a) were wounded at node 0 [local leaf; hereafter leaf 0 , and leaves $\mathrm{X}$ were used for naming the leaves at node $\mathrm{X}$ ( $\mathrm{X}$ represents the node number)], which was the second fully expanded leaf, and $20 \mu \mathrm{l}$ of $1 / 5$-diluted $M$. sexta OS were applied to the wounds (W+OS) to simulate $M$. sexta herbivory. JA and JA-Ile levels in local and systemic leaves were determined using a HPLC-MS/MS method. In the treated leaves, JA and JA-Ile levels increased after $10 \mathrm{~min}$, the levels were highest $1 \mathrm{~h}$ after the treatments, and decreased to almost the basal levels $2.5 \mathrm{~h}$ after induction (Figure $1 \mathrm{~b}$ ). In contrast, JA and JAIle levels in systemic leaves showed a very distinct pattern. JA accumulated almost exclusively in leaves +3 , with the highest levels 90 min after elicitation, whereas the other systemic leaves contained only minor amounts (Figure 1c). Importantly, the JA levels in leaves +3 were remarkable high: at $90 \mathrm{~min}$ after $\mathrm{W}+\mathrm{OS}$ treatment, JA contents reached up to $6 \mu \mathrm{g} \mathrm{g}^{-1}$ fresh mass (FM) JA, which were more than twice as much as the highest JA levels detected in the local leaves. The systemic distribution of JA-Ile was similar to that of JA but the highest levels in leaves +3 did not exceed those detected in local leaves, although $90 \mathrm{~min}$ after $\mathrm{W}+\mathrm{OS}$ treatment JA-Ile contents in leaves +3 were also 2 -fold greater than those in local leaves (Figure 1c). To determine whether systemic JA accumulations were limited to younger leaves, we elicited leaves 0 with $\mathrm{W}+\mathrm{OS}$ and quantified JA and JA-Ile levels in leaves -4 (4 positions older than the elicited leaf) to leaves +4 (the youngest leaf) 90 min after 
a
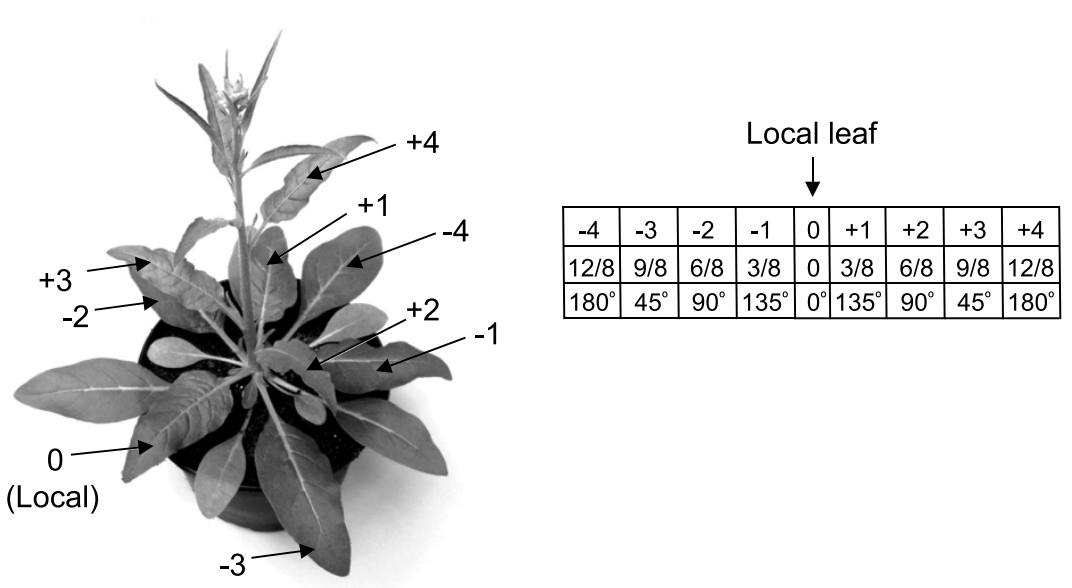

b
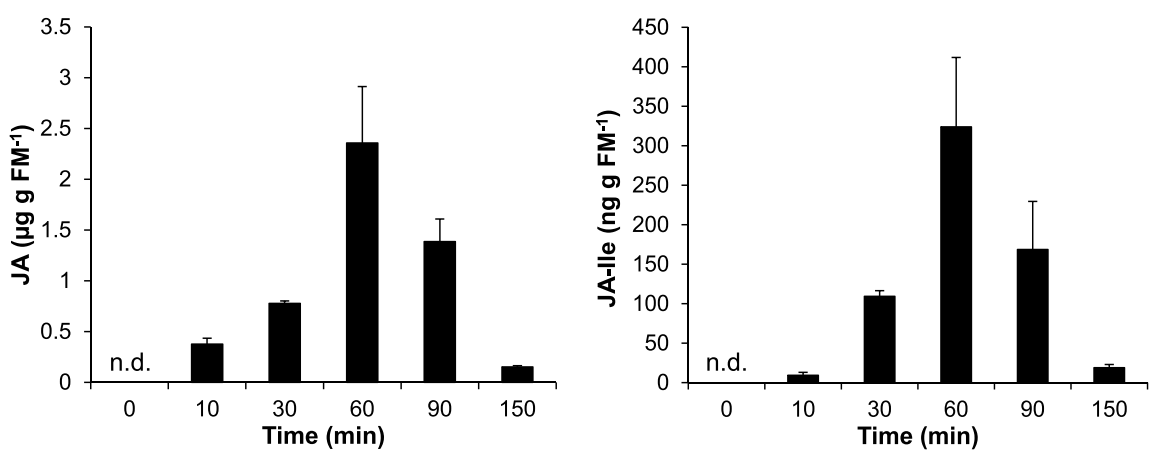

C
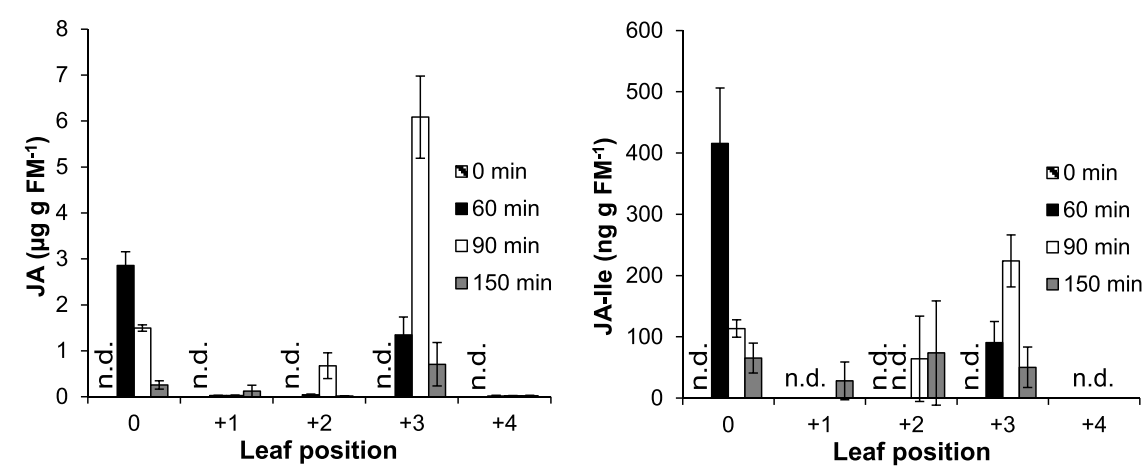

d
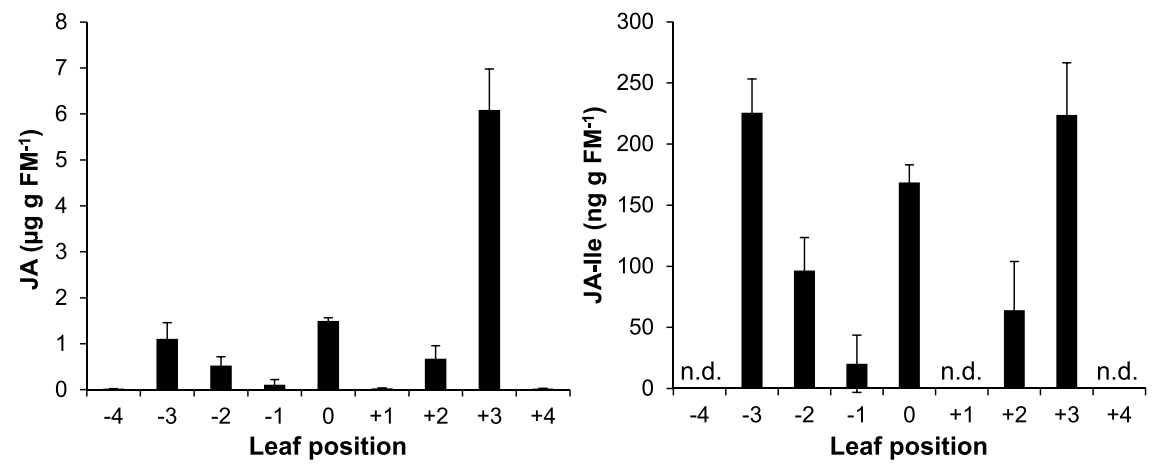

Figure 1 (See legend on next page.) 
Figure $1 \mathrm{~W}+$ OS-induced JA accumulation in local and systemic leaves. The leaves undergoing source-sink transition, designated as the leaves 0 , were wounded with a pattern wheel and $20 \mu \mathrm{l}$ of $1 / 5$ diluted $M$. sexta OS were immediately applied to wounds (W + OS). Samples were harvested at indicated times, and their JA and JA-lle contents were analyzed. a Numbering of the leaf positions in a bolting $N$. attenuata plant and the additive angular distances assuming $3 / 8$ phyllotaxis proceeding from the leaf 0 . $\mathbf{b} \mathrm{JA}$ and JA-lle JA levels in treated local leaves 0 . $\mathbf{c} \mathrm{JA}$ and JA-lle accumulation in local leaves 0 and in younger systemic leaves at different times after $\mathrm{W}+\mathrm{OS}$ treatment. $\mathbf{d} \mathrm{JA}$ and JA-lle levels in local and systemic leaves 90 min after $\mathrm{W}+\mathrm{OS}$ treatment. Values are mean $\pm \mathrm{SE} ; \mathrm{N}=5$; n.d. = not detectable.

$\mathrm{W}+$ OS elicitation (Figure 1d). Again, leaves +3 accumulated high JA levels (about $6.5 \mu \mathrm{g} \mathrm{g}^{-1} \mathrm{FM}$ ), but increased contents of JA could also be detected in leaves $-3-2$, and +2 , with $1,0.45$, and $0.4 \mu \mathrm{g} \mathrm{g}^{-1} \mathrm{FM}$, respectively (Figure 1d). Remarkably, these leaves accumulated relative high amounts of JA-Ile: leaves -3 contained $225 \mathrm{ng} \mathrm{g}^{-1} \mathrm{FM}$, as did leaves +3 (Figure 1d).

Thus, simulated $M$. sexta herbivory highly increases the accumulations of JA in local and systemic leaves, but systemically induced JA levels follow a pattern with very different increased levels in different leaves.

\section{Both wounding and FACs are required for systemic JA accumulation}

In maize leaves, wounding induces JA accumulation only at the immediate site of damage, whereas insect elicitors also induce JA accumulation in distant tissues [28]. Previous research on $N$. attenuata revealed that after simulated herbivory, JA levels in distal leaves accumulate to less than $10 \%$ of the local maximum $[9,23,24]$, and after wounding alone no increase in JA was detected [9]. To gain insight into the responses of systemic leaves to mechanical wounding, leaves 0 were wounded and $20 \mu \mathrm{l}$ of water were applied $(\mathrm{W}+\mathrm{W})$, and JA and JA-Ile accumulations were determined in all leaves. Local JA levels increased $10 \mathrm{~min}$ after the treatment and reached high values between 30 and 90 min (about $550 \mathrm{ng} \mathrm{g}^{-1} \mathrm{FM}$ ), which were significantly smaller than those detected after $\mathrm{W}+\mathrm{OS}$ elicitation; after $150 \mathrm{~min}$, JA levels decreased back to almost basal levels (Figure 2a). JA-Ile followed the JA pattern but accumulated to the highest levels after $30 \mathrm{~min}$ (137 $\mathrm{ng} \mathrm{g}^{-1} \mathrm{FM}$ ) (Figure 2a). In contrast to $\mathrm{W}+\mathrm{OS}$, $\mathrm{W}+\mathrm{W}$ treatment did not lead to detectably increased JA and JA-Ile levels in any systemic leaves 90 min after treatment (Figure 2b) and other times examined (30, 60, and $150 \mathrm{~min}$; these data are not shown). These findings suggest that the systemic JA accumulation is OS-dependent and wounding alone has non-detectable effect on systemic JA levels. FACs in $M$. sexta OS are known to be the elicitors for OS-specific plant responses, such as MAPK activation [3], JA burst, and accumulation of defense metabolites $[17,29]$. Given that applying OS to wounds (W + OS) induced systemic JA accumulation, we next explored whether FACs were responsible for this systemic response.

Twenty microliter of $\mathrm{N}$-linolenoyl-L-Glu, one of the most abundant FACs in $M$. sexta OS [17], at $27.6 \mathrm{ng} / \mu \mathrm{l}$ (similar to its concentration in 1/5 diluted OS), were applied to freshly wounded $N$. attenuata leaves 0 (W + FAC) and leaf samples were harvested $90 \mathrm{~min}$ after the treatment when systemic hormone levels attained the highest values (Figure 1c); in parallel, we applied FAC-free OS to wounds $(\mathrm{W}+\mathrm{FAC}$-free $\mathrm{OS})$. The $\mathrm{W}+\mathrm{FAC}$-induced JA and JA-Ile levels in local and systemic leaves largely resembled those after $\mathrm{W}+\mathrm{OS}$ treatment (Figure 2c), and similar to $\mathrm{W}+\mathrm{W}$, FAC-free OS highly induced JA in the local tissue but systemic JA remained below the detection limit, indicating that FACs are necessary to elicit systemic JA (Figure 2d). To exclude the possibility that FACs were transported from local to distal leaves through the plant vascular system and thus induced systemic JA accumulation, we pressure-inoculated $100 \mu \mathrm{l}$ of a FAC solution $(27.6 \mathrm{ng} / \mu \mathrm{l})$ into leaves 0 and measured local and systemic (leaves +3) JA levels after $90 \mathrm{~min}$. The solvent for the FAC $(0.05 \%$ Tween 20$)$ was inoculated as the control treatment, and it did not induce local or systemic JA (data not shown). In contrast, FAC-inoculated leaves accumulated $4.6 \mu \mathrm{g} \mathrm{g}^{-1} \mathrm{FM} \mathrm{JA}$, almost 3 times as much as the induced JA levels after $W+F A C$ treatments (Figure 2e), and importantly, FAC inoculation did not induce a strong accumulation of JA in systemic leaves, unlike what we saw in $\mathrm{W}+$ OS-treated plants (Figure 2e).

These data indicate that both FACs and wounding are required to induce a systemic signal that leads to JA accumulation in distal leaves.

\section{Simulated herbivory treatment induces MAPK activity in systemic leaves}

MAPKs are an essential part of the signaling cascade induced by wounding or herbivore attack. In tomato, wounding activates MAPKs both locally and systemically [18]. Wounding tobacco leaves with carborundum quickly increases the levels of WIPK (wound-induced protein kinase) transcripts in systemic leaves, and cutting tobacco stem activates WIPK systemically [19]. FACs are strong elicitors that amplify wounding-induced MAPK activation and potentiate the elicited JA burst in $N$. attenuata $[3,17]$. To explore whether systemic JA accumulation was correlated with increased MAPK activity in these tissues, we performed a series of in-gel MAPK activity assays. The basal SIPK activity in uninduced plants was similarly very low in all leaves (Additional file 1 ). Following $\mathrm{W}+\mathrm{OS}$ induction in leaves 0 , SIPK activity increased 

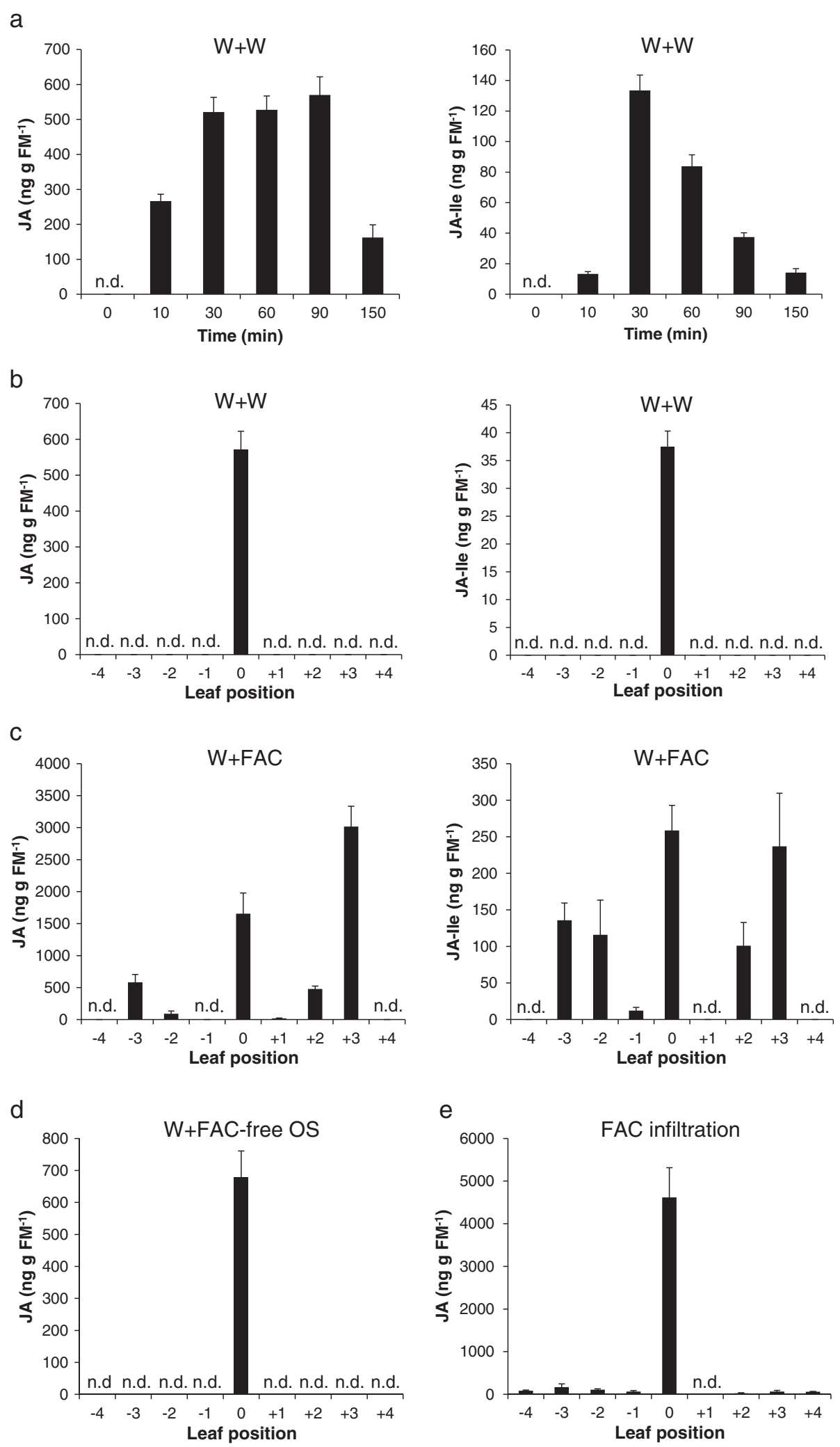

Figure 2 (See legend on next page.) 
(See figure on previous page.)

Figure 2 Wounding and FACs are both required for systemic JA accumulation. a JA levels in local leaves 0 after wounding. The leaves 0 were wounded with a pattern wheel and $20 \mu \mathrm{l}$ of water were immediately applied to wounds $(W+W)$, and the JA contents were analyzed. $\mathbf{b} \mathrm{JA}$ and JA-lle contents in local and systemic leaves 90 min after W + W treatment. $\mathbf{c}$ and $\mathbf{d}$ JA and JA-lle values in different leaves 90 min after applying FAC or FAC-free OS to wounds. Leaves 0 were wounded with a pattern wheel and $20 \mu \mathrm{l}$ of FAC (27.6 ng/ $\mu \mathrm{l} ; \mathrm{W}+\mathrm{FAC})$ or $20 \mu \mathrm{l}$ FAC-free M. sexta OS (W + FAC-free OS) were immediately applied to wounds. e JA contents in different leaves 90 min after pressure infiltration of $100 \mu \mathrm{l}$ FAC $(27.6 \mathrm{ng} / \mu \mathrm{l})$ into leaves 0 . Values are mean $\pm \mathrm{SE} ; \mathrm{N}=5$; n.d. $=$ not detectable.

locally and systemically and the distribution of SIPK activity levels in different leaves greatly resembled that of JA levels in these leaves (Figure 3a). Silencing SIPK highly compromises herbivory-induced JA accumulation [3], and these data suggest that SIPK activity might also be required for systemic JA induction: SIPK activity was the highest in the treated local leaves but about $50 \%$ less in leaves +3 , and leaves -3 and -2 had activity levels slightly above those in controls (Additional file 1). To gain further insight into the regulation of systemic kinase activation, we performed a time course experiment and compared kinase activity in local and the systemic leaves +3 . In treated leaves SIPK activity rapidly increased within $10 \mathrm{~min}$, peaked at $30 \mathrm{~min}$, and remained high until $90 \mathrm{~min}$ after induction (Figure 3b). In contrast, increase of SIPK activity in leaves +3 was not detected until $30 \mathrm{~min}$, and was very transient with a maximum at $60 \mathrm{~min}$ after the treatment (Figure $3 \mathrm{~b})$. This delayed MAPK response and subsequent JA accumulation in systemic leaves might reflect the time that the mobile signal(s) needs to travel from the treated leaves to the distal ones.

Consistent with the lack of elevated JA levels, $\mathrm{W}+\mathrm{W}$ did not increase systemic SIPK activity (Figure 3c). A more detailed analysis of the kinetic of SIPK activation also revealed no strong increase of SIPK activity in the leaves +3 (Figure 3d).

We conclude that after $M$. sexta herbivory, but not wounding, a mobile signal is rapidly propagated from damaged leaves to specific systemic leaves to induce MAPK signaling, and activation of MAPKs likely further triggers JA biosynthesis.

\section{Systemic induction of trypsin protease inhibitors does not require increased MAPK activity or JA contents in systemic leaves}

$M$. sexta attack increases the levels of TPI transcripts and activity in $N$. attenuata. This response is not limited to attacked leaves but spreads to systemic ones [30,31]. TPI expression is dependent on JA signaling as COI1- and LOX3-silenced plants that are defective in JA perception and production, respectively, have very little TPI activity and do not accumulate TPI after W+OS elicitation [32,33]. To investigate the pattern of TPI activity in local and systemic leaves and to reveal whether it is correlated with MAPK activation and induced JA/JA-Ile levels, we treated leaves 0 with $\mathrm{W}+\mathrm{W}$ and $\mathrm{W}+\mathrm{OS}$ and analyzed

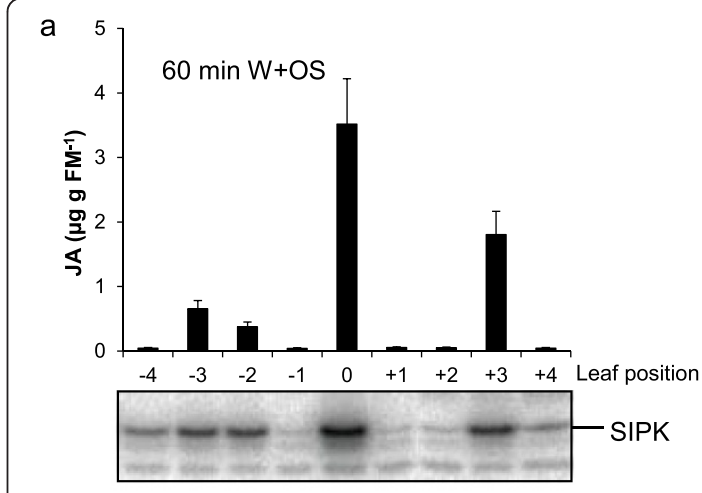

b

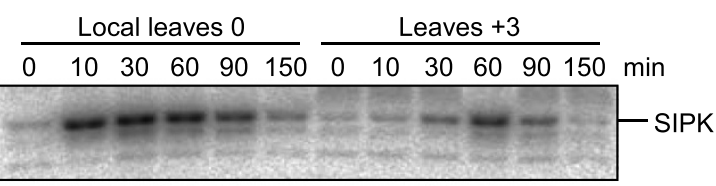

C

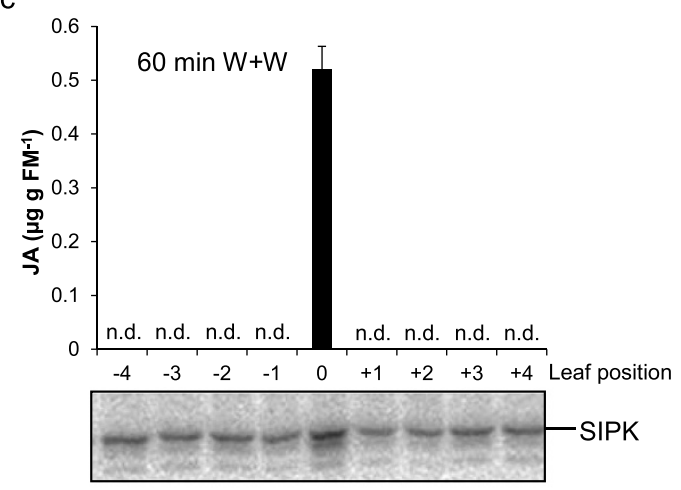

d

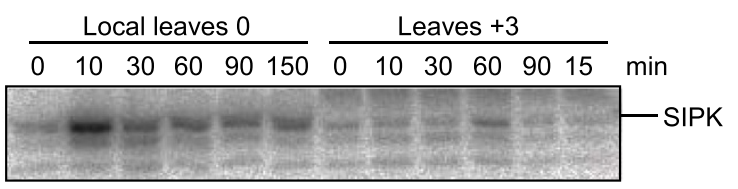

Figure 3 SIPK activation and JA accumulation show similar leaf distribution. The leaves 0 were wounded with a pattern wheel and $20 \mu \mathrm{l}$ of $1 / 5$ diluted $M$. sexta OS ( $\mathbf{a}$ and $\mathbf{b}$ ) or water ( $\mathbf{c}$ and $\mathbf{d}$ ) were immediately applied to wounds (W + OS). Local and systemic leaves $(\mathrm{N}=5)$ were harvested at indicated times and JA contents (mean $\pm \mathrm{SE}$ ) were analyzed; SIPK activity was analyzed in pooled samples using an in-gel kinase assay. 
TPI activity in local and systemic leaves 3 days after elicitation. Without treatment, highest TPI levels were found in young leaves, and the older ones had very low TPI activity (Figure 4). Wounding elicited increased TPI activity levels in leaves 0 and in leaves +2 and +3 , with values 2- to 3 -fold of those in uninduced respective controls. In contrast, $\mathrm{W}+\mathrm{OS}$ treatment induced TPI levels in almost all leaves (Figure 4). Similar to W $+\mathrm{W}$ treatment, highest values were detected in the local leaves 0 and in leaves +2 and +3 , whose TPI activity levels were twice as much as those induced by wounding; remarkably, despite relatively high $\mathrm{W}+$ OS-induced JA-Ile levels in systemic leaves -3 and -2 (Figure 1d), these leaves exhibited only minor TPI activity (Figure 4).

We supposed that older leaves might have decreased inducibility of TPI after elicitation of JA. To examine the inducibility of TPI in different leaves in response to jasmonate elicitation, methyl jasmonate was applied to leaves at all positions on individual plants and TPI activity was quantified after 3 days. TPI activity levels were lowest in the oldest leaves -4 , increased in younger leaves, and the youngest leaves +4 had about 3.6 times more TPI activity than leaves -4 (Additional file 2), confirming that JAinduced TPI levels decrease with increasing leaf age.

Therefore, unlike wounding, simulated $M$. sexta feeding induces increase of TPI activity in almost all leaves, although systemic TPI activity increases more strongly in younger leaves. Importantly, systemic leaves that have highly induced TPI activity do not necessarily have elevated MAPK activity and JA contents.

\section{Rapid mobile long-distance signals induce systemic defense responses}

The increased MAPK activity, JA levels, and TPI activity in systemic leaves after $\mathrm{W}+\mathrm{OS}$ elicitation revealed that

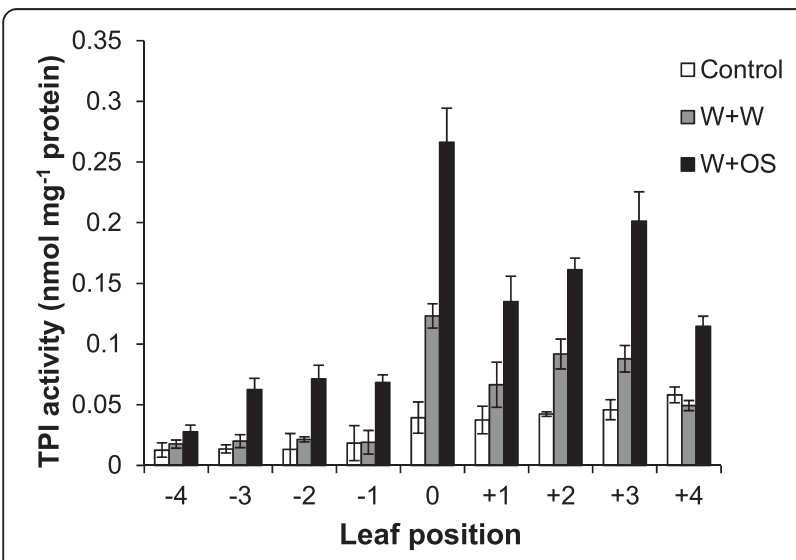

Figure 4 TPI activity in local and systemic leaves. Leaves 0 were wounded with a pattern wheel and $20 \mu$ of water $(W+W)$ or $M$. sexta OS (W+ OS) were applied to the wounds. Treated leaves and systemic leaves were harvested 3 days after the treatment and TPI activity was analyzed. Values are mean $\pm \mathrm{SE} ; \mathrm{N}=5$. certain long-distance signals are propagated from local leaves to systemic ones to activate these responses. To estimate the time required for the TPI-inducing systemic signal to exit from the wounded leaf, we treated leaves 0 with $\mathrm{W}+\mathrm{OS}$ and then removed them (by excising from petioles) at $0,1,5$, and 10 min after the treatments; untreated plants and plants treated with $\mathrm{W}+\mathrm{OS}$ whose local leaves were retained were used for comparisons. Three days after these initial treatments, TPI activity was measured in systemic leaves (Figure $5 \mathrm{a}$ ). Immediately removing the treated leaves did not induce any changes of TPI activity, and similarly, excision of the damaged leaves in 1 or $5 \mathrm{~min}$ also induced very little systemic TPI (Figure 5a). However, when the local leaves were removed $10 \mathrm{~min}$ after the treatment, TPI activity levels in systemic leaves almost fully elevated to those in plants whose treated leaves were retained (Figure 5a). These results suggest that systemic TPI induction involves a signal that exits the wounded leaves between 5 to $10 \mathrm{~min}$, and given that the petiole lengths are about $3 \mathrm{~cm}$, the speed of the signal traveling out of the treated leaves is approximately $0.3 \mathrm{~cm} / \mathrm{min}$. These findings are consistent with an earlier study in $N$. attenuata where it was shown that removing a 3-mm-wide zone adjacent to the $\mathrm{W}+\mathrm{OS}$ treatment site within $40 \mathrm{~s}$ did not prevent the induction of JA in the remaining leaf tissue [34].

To investigate how fast the signal that triggers MAPK activation and JA accumulation travels out of herbivoredamaged leaves, we excised $\mathrm{W}+\mathrm{OS}$-elicited leaves at different times after the treatment and measured JA accumulation in leaves +3 after 90 min when JA contents reach the highest values. Leaf excision alone did not increase systemic JA levels (Figure 5b), and excising the local leaves $10 \mathrm{~min}$ after treatment resulted in 30\% increased JA contents; excising the local leaves $15 \mathrm{~min}$ after the treatment almost fully elicited JA levels in leaves +3 (Figure 5b). Likewise, leaves +3 from plants whose local leaves were ablated 10 min after $\mathrm{W}+\mathrm{OS}$ treatment showed similar SIPK activity levels as those whose local leaves were retained (Figure 5c). It seems that the speed of this signal is not very different from that of the signal activating systemic TPI.

\section{Discussion}

Herbivore feeding induces plant defense responses not only in the local attacked leaves, but also in distal undamaged ones. How plants regulate these defense responses is still poorly understood. Here we demonstrate that $M$. sexta OS applied to wounds elicits systemic induction of MAPK activity and JA accumulation. Our results suggest that $N$. attenuata is able to recognize herbivore feeding by perceiving FACs penetrated into wounds and deploying specific responses in undamaged 


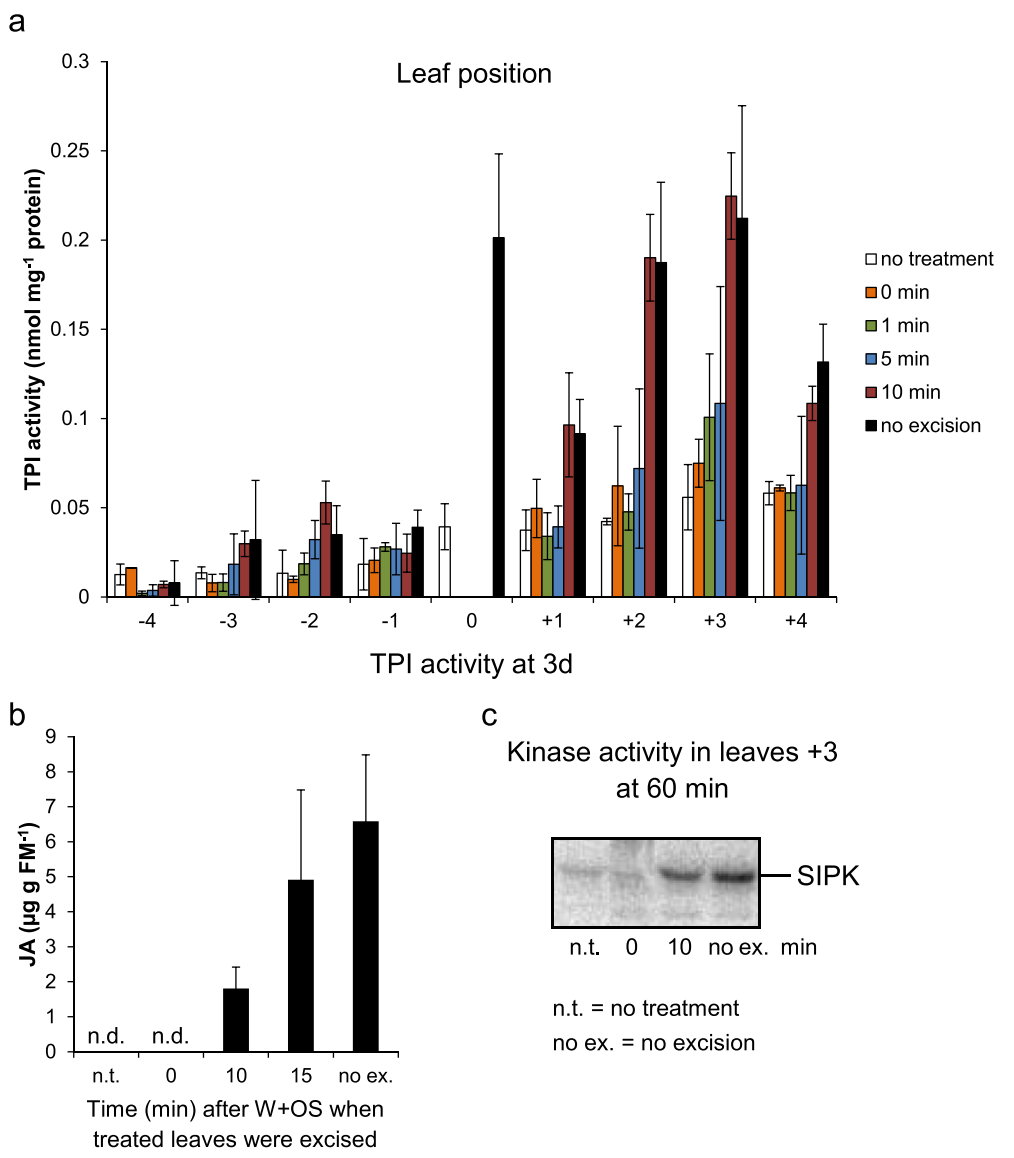

Figure 5 Systemic responses after W + OS elicitation and leaf excision. Local leaves 0 were W + OS-elicited, and these leaves including petioles were ablated at indicated times after treatment and the elicited systemic responses were determined. a TPI activity (mean \pm SE, $N=5$ ) in different leaves, $3 \mathrm{~d}$ after elicitation of leaves 0 , which were either not excised or ablated at different times [untreated plants ("no treatment") served as comparisons]. b JA accumulation (mean $\pm \mathrm{SE}, \mathrm{N}=5$ ) in leaves $+3,90$ min after local leaves were elicited with $\mathrm{W}+\mathrm{OS}$ and ablated at indicated times. c SIPK activity in systemic leaves $+3,60$ min after the leaves 0 were treated with $W+O S$.

systemic leaves, including MAPK activation, JA accumulation, and later, increased TPI activity.

\section{Herbivory but not wounding elicits early systemic responses}

Studies on $N$. tabacum revealed $3 / 8$ phyllotaxis for rosette stage plants and $5 / 13$ phyllotaxis for the stem of elongated plants [35-37]. Accordingly, the systemic leaves analyzed in the present study were not directly vascular connected with the treated leaves but grew in specific angular distances resulting in different transvascular resistance levels $[30,38]$. As the transvascular resistance is often higher than the axial resistance, especially when the stems are relatively short [38], the angular distances between the treated leaves and systemic leaves may significantly influence the systemic signaling. In tomato, the intensity of systemic TPI accumulation was found to correlate with the degree of vascular linkage between and within leaves $[39,40]$. The same is true for salicylic acid transport in $N$. tabacum [41]. We detected highest JA levels in leaves +3 , which have together with leaves -3 the smallest angular distance (45 degree) to the local leaves at node 0 (Figure 1a and d). Also leaves +2 and -2 , with a shift of about 90 degrees to node 0 , had significantly increased JA levels 90 min after W + OS (Figure 1d). In contrast, leaves +1 and -1 with about 135 degree, and +4 and -4 leaves with about 180 degree angles to the local leaves did not show increased JA levels even $150 \mathrm{~min}$ after elicitation (Figure 1c). Clearly, the angular distance between local and systemic leaves is important in determining the levels of JA in those leaves and the elicited JA contents decrease with increasing angles.

Several other studies conducted on $N$. attenuata revealed only minor systemic JA concentrations, which were about $5-10 \%$ of the locally induced JA levels $[9,23,24,42]$. However, our comprehensive analysis indicated that systemic responses depend on leaf positions and the time after treatment. Furthermore, in Arabidopsis and Solanum nigrum, systemic JA levels 
also increase to only $10 \%$ of the local values [21,22,43], implying that systemic defense signaling might be species specific. It was found that $N$. attenuata systemic leaves -1 do not have elevated SIPK activity after simulated $M$. sexta herbivory treatment was applied to leaves +1 [3]; however, this comprehensive study pointed out that after W+OS treatment, some systemic leaves do have highly elevated SIPK activity, and the previously proposed model should be updated.

Two lines of evidence support the notion that highly elevated systemic JA levels are unlikely to be transported from the damaged leaves to the systemic ones, but JA is de novo synthesized in the systemic leaves: Firstly, $\mathrm{W}+$ OS-induced JA levels in leaves +3 even exceeded those in the local leaves. Secondly, our leaf ablation experiments revealed that $10 \mathrm{~min}$ after local induction, the systemic signal had left the treated leaves and at this time point $\mathrm{W}+\mathrm{W}$ and $\mathrm{W}+\mathrm{OS}$ treatment elicited similar amounts of JA in local leaves (Figure $1 \mathrm{~b}$ and $2 \mathrm{a}$ ) but only $\mathrm{W}+\mathrm{OS}$ induced systemic JA accumulation. These findings are also supported by the studies in $N$. attenuata and Arabidopsis that JA-Ile and MeJA are de novo synthesized in systemic leaves, not transported from the wounded leaves $[9,22,23]$.

In Arabidopsis, wounding is sufficient to elevate systemic JA levels [21,22], but in Zea mays, Solanum nigrum, and $N$. attenuata, wounding alone induces JA accumulation only at the adjacent site of damage, whereas insect elicitors induce JA accumulation in distant tissues $[9,28,44]$. Similarly, systemic MAPK activation after wounding has been reported in some plant species, including soybean, tomato, and tobacco $[18,19,45]$; but wounding alone failed to induce systemic MAPK activity in $N$. attenuata (this study). By adding FACs to wounds and by removing them from OS, we show that FACs are the elicitors of the systemic JA response; however, FACs themselves appeared not to be the systemic signal, given that 1) FACs are quickly degraded after entering plant tissue [44], and 2) inoculating FACs into local tissue did not elicit JA responses in distal leaves (Figure 2e). The reason why FACs require wounding for activating systemic JA accumulation remains unknown, but it is possible that wounds are necessary for efficient loading of FACs to mechanically broken vascular tissues. During infiltration, FACs may remain in the apoplast and could not be transported to systemic leaves. Radio-traceable FACs could be used for elucidating whether FACs can be transported to systemic leaves.

These findings strongly suggest that a rapid mobile signal, which is elicited by FACs penetrated into wounds, but not by wounding alone, activates SIPK, and thereafter, SIPK activates JA biosynthesis in systemic leaves.

\section{Herbivory, but not wounding, strongly activates the late systemic response, TPI accumulation}

We found that unlike SIPK and JA, which were activated only in specific systemic leaves, simulated herbivory elicited the accumulation of TPI in all systemic leaves tested, but wounding only elevated TPI levels in systemic leaves +2 and +3 . The different distribution between early (SIPK and JA) and late (TPI) responses argues that the signal that triggers systemic TPI accumulation is likely different from the one that activates SIPK and initiates JA biosynthesis, and systemically increased JA levels are not important for elevation of TPI activity. Alternatively, the systemic leaf +3 with very pronounced JA accumulation (and MAPK activity) could serve as a "hub" for jasmonate distribution throughout the plant by inducing leaves in close phyllotactic positions and other distal leaves. Moreover, it cannot be excluded that TPI protein itself is re-distributed within the whole plant and thus also accumulates in leaves without a previous JA induction. These possibilities should be examined further.

The biological significance of the specific spatial distribution of systemic SIPK and JA remains unknown. We hypothesize that certain defense responses, such as terpenoids, some of which are known to have a function as indirect defenses [46], downstream of these signaling factors are also specifically mounted in these systemic leaves, and furthermore, systemic activation of SIPK and JA may also induce transmissible signals to other parts of the plant to further propagate or strengthen systemic defenses (metabolites).

In tomato, grafting of mutants deficient in JA production and perception indicated that induction of systemic TPI requires both the biosynthesis of jasmonic acid at the site of wounding and the ability to perceive a jasmonate signal in systemic leaves, but JA biosynthesis in systemic leaves and JA perception in local ones are not important for systemic TPI induction [47,48]. Our leaf ablation experiments showed that the signal released within 10 min after $\mathrm{W}+$ OS treatment from local leaves almost fully induced TPI activity in systemic leaves; furthermore, within $10 \mathrm{~min}$, local JA levels only elevated to about $10 \%$ of the highest JA levels produced, and these were similar in $\mathrm{W}+\mathrm{W}$ and $\mathrm{W}+\mathrm{OS}$ treated leaves (Figures $1 \mathrm{~b}$ and $5 \mathrm{~b}$ ). Thus, these data suggest that JA levels induced in local leaves are not directly involved in controlling systemic TPI accumulation. We propose that a signal induced by FACs is transported to systemic leaves, and there together with JA signaling (but not JA biosynthesis), induces TPI production. This intriguing observation clearly deserves more attention.

\section{The nature of the mobile signals}

Several studies suggest the involvement of hydraulic or electric signals in systemic signaling [22,49-52]. Given 
that our treatments $\mathrm{W}+\mathrm{W}$ and $\mathrm{W}+\mathrm{OS}$ likely generate similar hydraulic pressures to the systemic tissues, the hypothesis that hydraulic pressure is the only mobile signal can be ruled out. In lima bean (Phaseolus lunatus), FACs, but not wounding alone, specifically induce changes of cell membrane polarization [53]. Recent data from Arabidopsis indicate that wounding activates surface potential changes and experimental current injection into leaves leads to activation of JA biosynthesis and transcriptome changes [54]. It would be valuable to examine the changes of surface potentials of $N$. attenuata in local and systemic leaves. In $N$. attenuata, the signal that induces systemic SIPK and JA accumulation exits the treated leaves at about $0.3 \mathrm{~cm} / \mathrm{min}$; in Arabidopsis, the speed of the mobile signal, which induces JA-Ile accumulation in systemic leaves, is about $2 \mathrm{~cm} / \mathrm{min}$ [22]; in contrast, in Solanum nigrum, the mobile signal that elicits the systemic defensive compound, leucine aminopeptidase, needs much longer time - 90 to $240 \mathrm{~min}$ to exit the local leaves [44]. Elucidating the nature of the mobile signals in different species will also shed light on the large variations of the speeds of these signal transmissions.

In addition to TPIs, nicotine, and terpene-derived volatiles serve as important herbivory-inducible systemic defenses in $N$. attenuata [55-57]. Given that very likely different mobile signals induce systemic accumulation of JA (and activation of SIPK) and TPI, possibly other types of mobile signals are responsible for activating other systemic defenses; for example, recently, it was found that in $N$. attenuata JA perception and synthesis are important for wounding-induced putrescine methyltransferase transcript levels in roots and for the transport of de novo synthesized nicotine to leaves, implying that the regulation of root nicotine is modulated by a pathway different from the one that controls systemic TPI [58]. Transcriptome rearrangements and metabolite accumulations have also been observed in systemic leaves in other species, such as Arabidopsis, tomato, poplar, and soybean $[6,22,59,60]$. The identities of the transmissible signals, whether they are similar or speciesspecific, and how they are transported and function, and importantly, the ecological function of systemic defense are all very interesting questions to explore.

\section{Conclusions}

This study comprehensively demonstrates how plants respond to leaf herbivory on multiple levels, including signaling and defensive metabolite accumulation in local and systemic leaves, and highlights the importance of insect-derived elicitors in plant systemic defenses.

\section{Methods}

Plant growth and sample treatments

Nicotiana attenuata Torr. Ex W. (originally collected from the DI ranch, Santa Clara, UT) (Solanaceae) seeds were from an inbred line maintained in the Baldwin laboratory, and the seeds can be distributed by I.T. Baldwin, Max Planck Institute for Chemical Ecology, upon request. Voucher specimens of $N$. attenuata can be accessed at the Cornell University Herbarium (1989, I.T. Baldwin).

Seed germination and plant cultivation followed Krügel et al. [61]. Seeds were germinated on Petri dishes to synchronize their germination, and the seedlings were transferred to soil after 10 days. Four- to 5-week-old plants were used for all experiments.

For collection of $M$. sexta oral secretions (OS), $M$. sexta larvae were reared on $N$. attenuata plants until the third to fifth instars. OS were collected on ice as described in Roda et al. [62] and stored under nitrogen at $-20^{\circ} \mathrm{C}$. For simulated herbivory treatment, leaves at position 0 were wounded with a pattern wheel and 1/5 diluted OS were immediately rubbed onto each wounded leaf (W + OS); for wounding treatment, leaves were wounded with a pattern wheel, and $20 \mu \mathrm{l}$ of water were rubbed onto each leaf $(W+W)$. MeJA (methyl jasmonate) was dissolved in heat-liquefied lanolin at a concentration of $7.5 \mathrm{mg} / \mathrm{ml} ; 20 \mu \mathrm{l}$ of the resulting lanolin paste was applied to the base of the leaves, and pure lanolin was applied as a control. FAC (N-linolenoyl-L-Glu) was synthesized inhouse [17], which was dissolved in $0.05 \%$ Tween 20 at a concentration of $27.6 \mathrm{ng} / \mu \mathrm{l}$ (similar to that in $1 / 5$ diluted OS). FAC-free OS was prepared by passing OS four times through spin columns filled with Amberlite IRA-400 resin (Sigma-Aldrich) [17]. Twenty microliters of each test solution were applied to each leaf. After specific times, leaves were excised, immediately frozen in liquid nitrogen, and stored at $-80^{\circ} \mathrm{C}$ until use.

\section{Analysis of JA and JA-lle concentrations}

One milliliter of ethyl acetate spiked with $200 \mathrm{ng}$ of $\mathrm{D}_{2}-\mathrm{JA}$ and $40 \mathrm{ng}$ of ${ }^{13} \mathrm{C}_{6}$-JA-Ile, the internal standards for JA and JA-Ile, respectively, was added to each briefly crushed leaf sample ( 150 mg). Samples were then ground on a FastPrep homogenizer (Thermo Electron). After being centrifuged at $13,000 \mathrm{~g}$ for $10 \mathrm{~min}$ at $4{ }^{\circ} \mathrm{C}$, supernatants were transferred to fresh tubes and evaporated to dryness on a vacuum concentrator (Eppendorf). Each residue was resuspended in $0.5 \mathrm{ml}$ of $70 \%$ methanol $(\mathrm{v} / \mathrm{v})$ and centrifuged at $13,000 \mathrm{~g}$ for $15 \mathrm{~min}$ at $4^{\circ} \mathrm{C}$ to remove particles. The supernatants were analyzed on a HPLC-MS/MS (LCMS8040, Shimadzu).

\section{In-gel kinase activity assay}

Leaf tissue pooled from 4 replicate leaves was crushed in liquid nitrogen, and $200 \mu \mathrm{l}$ of protein extraction buffer [100 mM HEPES, pH 7.5, 5 mM EDTA, 5 mM EGTA, $10 \mathrm{mM} \mathrm{Na}_{3} \mathrm{VO}_{4}, 10 \mathrm{mM} \mathrm{NaF}, 50 \mathrm{mM} \beta$-glycerolphosphate, $1 \mathrm{mM}$ phenylmethylsulfonyl fluoride, $10 \%$ glycerol, and EDTA-free proteinase inhibitor cocktail (Roche 
Diagnostics)] was added to $\sim 100 \mathrm{mg}$ of tissue. Leaf tissue was then completely suspended by vortexing. After being centrifuged at $4^{\circ} \mathrm{C}$ at maximum speed for $20 \mathrm{~min}$, supernatants were transferred to fresh tubes. Protein concentrations were measured using a Bio-Rad protein assay kit with bovine serum albumin as a standard. In-gel MAPK activity assays were done following Zhang \& Klessig [63] using myelin basic protein (MBP) as the substrate. Gel images were obtained on an FLA-3000 phosphor imager system (Fujifilm).

\section{Analyses of TPI activity}

TPI activity was analyzed with a radial diffusion assay described by van Dam et al. [31].

\section{Availability of supporting data}

The data sets supporting the results of this article are included within the article and its additional files.

\section{Additional files}

Additional file 1: MAPK activity in uninduced leaves. Kinase activity was analyzed in pooled samples of 5 replicated leaves of untreated plants by an in-gel kinase assay using myelin basic protein (MBP) as the substrate. Numbers above the gel image indicate the leaf positions.

Additional file 2: Methyl jasmonate treatment induces higher TPI activity in younger leaves. MeJA was dissolved in heat-liquefied lanolin at a concentration of $7.5 \mu \mathrm{g} \mathrm{l}^{-1} ; 20 \mu \mathrm{l}$ of the resulting lanolin paste was applied to leaves at individual plants, and TPI activity (mean \pm SE) was measured 3 days after the treatment $(\mathrm{N}=5)$.

\section{Competing interests}

The authors declare that they have no competing interests.

\section{Authors' contributions}

$\mathrm{CH}, \mathrm{MH}$ and JW participated in the design of the study. $\mathrm{CH}$ and $\mathrm{MH}$ performed the experimental work and analyzed the data. $\mathrm{CH}$, ITB and JW wrote the manuscript. All authors read and approved the final manuscript.

\section{Acknowledgements}

We thank Dr. Mario Kallenbach for synthesizing FACs. This work was funded by the Strategic Priority Research Program of the Chinese Academy of Sciences, Grant No. XDB11050200, the Max Planck Society, a grant from the Yunnan Talent Recruitment Program (Grant No. 2012HA016), and the 1000 Young Talent Recruitment

\section{Author details}

${ }^{1}$ Kunming Institute of Botany, Chinese Academy of Sciences, 650201 Kunming, China. ${ }^{2}$ Max Planck Institute for Chemical Ecology, Hans-Knoell Str. 8, 07745 Jena, Germany.

Received: 18 August 2014 Accepted: 6 November 2014

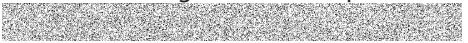

\section{References}

1. Mithofer A, Boland W: Recognition of herbivory-associated molecular patterns. Plant Physiol 2008, 146(3):825-831.

2. Hettenhausen C, Baldwin IT, Wu JQ: Nicotiana attenuata MPK4 suppresses a novel jasmonic acid (JA) signaling-independent defense pathway against the specialist insect Manduca sexta, but is not required for the resistance to the generalist Spodoptera littoralis. New Phytol 2013, 199(3):787-799.
3. Wu JQ, Hettenhausen C, Meldau S, Baldwin IT: Herbivory rapidly activates MAPK signaling in attacked and unattacked leaf regions but not between leaves of Nicotiana attenuata. Plant Cell 2007, 19(3):1096-1122.

4. Kandoth PK, Ranf S, Pancholi SS, Jayanty S, Walla MD, Miller W, Howe GA, Lincoln DE, Stratmann JW: Tomato MAPKs LeMPK1, LeMPK2, and LeMPK3 function in the systemin-mediated defense response against herbivorous insects. Proc Natl Acad Sci U S A 2007, 104(29):12205-12210.

5. Schmelz EA, Engelberth J, Alborn HT, Tumlinson JH, Teal PEA: Phytohormone-based activity mapping of insect herbivore-produced elicitors. Proc Natl Acad Sci U S A 2009, 106(2):653-657.

6. Green TR, Ryan CA: Wound-induced proteinase inhibitor in plant leaves possible defense mechanism against insects. Science 1972, 175(4023):776

7. Heidel AJ, Baldwin IT: Microarray analysis of salicylic acid- and jasmonic acid-signalling in responses of Nicotiana attenuata to attack by insects from multiple feeding guilds. Plant Cell Environ 2004, 27(11):1362-1373.

8. Kim SG, Yon F, Gaquerel E, Gulati J, Baldwin IT: Tissue Specific Diurnal Rhythms of Metabolites and Their Regulation during Herbivore Attack in a Native Tobacco, Nicotiana attenuata. Plos One 2011, 6(10):e26214

9. von Dahl CC, Baldwin IT: Methyl jasmonate and cis-jasmone do not dispose of the herbivore-induced jasmonate burst in Nicotiana attenuata. Physiol Plant 2004, 120(3):474-481.

10. Li Q, Xie QG, Smith-Becker J, Navarre DA, Kaloshian I: Mi-1-mediated aphid resistance involves salicylic acid and mitogen-activated protein kinase signaling cascades. Mol Plant Microbe In 2006, 19(6):655-664.

11. Howe GA, Jander G: Plant immunity to insect herbivores. Annu Rev Plant Biol 2008, 59:41-66.

12. Wasternack C: Jasmonates: an update on biosynthesis, signal transduction and action in plant stress response, growth and development. Ann Bot-London 2007, 100(4):681-697.

13. Kessler A, Halitschke R, Baldwin IT: Silencing the jasmonate cascade: induced plant defenses and insect populations. Science 2004, 305(5684):665-668.

14. Seo S, Okamoto M, Seto H, Ishizuka K, Sano H, Ohashi Y: Tobacco MAP kinase: a possible mediator in wound signal transduction pathways. Science 1995, 270(5244):1988-1992.

15. Ichimura K, Mizoguchi T, Yoshida R, Yuasa T, Shinozaki K: Various abiotic stresses rapidly activate Arabidopsis MAP kinases ATMPK4 and ATMPK6. Plant J 2000, 24(5):655-665.

16. Park JH, Halitschke R, Kim HB, Baldwin IT, Feldmann KA, Feyereisen R: A knock-out mutation in allene oxide synthase results in male sterility and defective wound signal transduction in Arabidopsis due to a block in jasmonic acid biosynthesis. Plant J 2002, 31(1):1-12.

17. Halitschke R, Schittko U, Pohnert G, Boland W, Baldwin IT: Molecular interactions between the specialist herbivore Manduca sexta (Lepidoptera, Sphingidae) and its natural host Nicotiana attenuata. III. Fatty acid-amino acid conjugates in herbivore oral secretions are necessary and sufficient for herbivore-specific plant responses. Plant Physiol 2001, 125(2):711-717.

18. Stratmann JW, Ryan CA: Myelin basic protein kinase activity in tomato leaves is induced systemically by wounding and increases in response to systemin and oligosaccharide elicitors. Proc Natl Acad Sci U S A 1997, 94(20):11085-11089.

19. Seo S, Sano H, Ohashi $Y$ : Jasmonate-based wound signal transduction requires activation of WIPK, a tobacco mitogen-activated protein kinase. Plant Cell 1999, 11(2):289-298.

20. Dombrowski JE, Hind SR, Martin RC, Stratmann JW: Wounding systemically activates a mitogen-activated protein kinase in forage and turf grasses. Plant Sci 2011, 180(5):686-693.

21. Glauser G, Grata E, Dubugnon L, Rudaz S, Farmer EE, Wolfender JL: Spatial and temporal dynamics of jasmonate synthesis and accumulation in Arabidopsis in response to wounding. J Biol Chem 2008, 283(24):16400-16407.

22. Koo AJK, Gao XL, Jones AD, Howe GA: A rapid wound signal activates the systemic synthesis of bioactive jasmonates in Arabidopsis. Plant J 2009, 59(6):974-986.

23. Wang L, Allmann S, Wu J, Baldwin IT: Comparisons of LIPOXYGENASE3- and JASMONATE-RESISTANT4/6-silenced plants reveal that jasmonic acid and jasmonic acid-amino acid conjugates play different roles in herbivore resistance of Nicotiana attenuata. Plant Physiol 2008, 146(3):904-915.

24. Stitz M, Gase K, Baldwin IT, Gaquerel E: Ectopic expression of AtJMT in Nicotiana attenuata: creating a metabolic sink has tissue-specific consequences for the jasmonate metabolic network and silences downstream gene expression. Plant Physiol 2011, 157(1):341-354. 
25. Wu JQ, Baldwin IT: New insights into plant responses to the attack from insect herbivores. Annu Rev Genet 2010, 44:1-24.

26. Baldwin IT: An ecologically motivated analysis of plant-herbivore interactions in native tobacco. Plant Physiol 2001, 127(4):1449-1458.

27. Browse J: Jasmonate passes muster: a receptor and targets for the defense hormone. Annu Rev Plant Biol 2009, 60:183-205.

28. Engelberth J, Seidl-Adams I, Schultz JC, Tumlinson JH: Insect elicitors and exposure to green leafy volatiles differentially upregulate major octadecanoids and transcripts of 12-oxo phytodienoic acid reductases in Zea mays. Mol Plant Microbe In 2007, 20(6):707-716.

29. Halitschke R, Gase K, Hui DQ, Schmidt DD, Baldwin IT: Molecular interactions between the specialist herbivore Manduca sexta (Lepidoptera, Sphingidae) and its natural host Nicotiana attenuata. VI. Microarray analysis reveals that most herbivore-specific transcriptional changes are mediated by fatty acid-amino acid conjugates. Plant Physiol 2003, 131(4):1894-1902.

30. Schittko U, Baldwin IT: Constraints to herbivore-induced systemic responses: bidirectional signaling along orthostichies in Nicotiana attenuata. J Chem Ecol 2003, 29(3):763-770.

31. van Dam NM, Horn M, Mares M, Baldwin IT: Ontogeny constrains systemic protease inhibitor response in Nicotiana attenuata. J Chem Ecol 2001, 27(3):547-568

32. Halitschke R, Baldwin IT: Antisense LOX expression increases herbivore performance by decreasing defense responses and inhibiting growth-related transcriptional reorganization in Nicotiana attenuata. Plant J 2003, 36(6):794-807.

33. Paschold A, Halitschke R, Baldwin IT: Co(i)-ordinating defenses: NaCOI1 mediates herbivore-induced resistance in Nicotiana attenuata and reveals the role of herbivore movement in avoiding defenses. Plant $J$ 2007, 51(1):79-91.

34. Schittko U, Preston CA, Baldwin IT: Eating the evidence? Manduca sexta larvae can not disrupt specific jasmonate induction in Nicotiana attenuata by rapid consumption. Planta 2000, 210(2):343-346.

35. Allard HA: Some aspects of the phyllotaxy of tobacco. J Agric Res 1942, 64:0049-0055.

36. Shirova M, Lister GR DNC, Krotov G: Translocation of C14 in tobacco at different stages of development following assimilation of $\mathrm{C} 14 \mathrm{O} 2$ by a single leaf. Can J Bot 1961, 39:855-864.

37. Jones H, Martin RV, PORTER HK: Translocation of 14carbon in tobacco following assimilation of 14carbon dioxide by a single leaf. Ann Bot London 1959, 23:493-510.

38. Fiscus EL, Parsons LR, Alberte RS: Phyllotaxy and water relations in tobacco. Planta 1973, 112(4):285-292.

39. Rhodes JD, Thain JF, Wildon DC: Evidence for physically distinct systemic signalling pathways in the wounded tomato plant. Ann Bot-London 1999, 84(1):109-116.

40. Orians CM, Pomerleau J, Ricco R: Vascular architecture generates fine scale variation in systemic induction of proteinase inhibitors in tomato. J Chem Ecol 2000, 26(2):471-485.

41. Shulaev V, Leon J, Raskin I: Is salicylic-acid a translocated signal of systemic acquired-resistance in tobacco. Plant Cell 1995, 7(10):1691-1701.

42. Pluskota WE, Qu N, Maitrejean M, Boland W, Baldwin IT: Jasmonates and its mimics differentially elicit systemic defence responses in Nicotiana attenuata. J Exp Bot 2007, 58(15-16):4071-4082.

43. VanDoorn A, Bonaventure G, Schmidt DD, Baldwin IT: Regulation of jasmonate metabolism and activation of systemic signaling in Solanum nigrum: COI1 and JAR4 play overlapping yet distinct roles. New Phytol 2011, 190(3):640-652.

44. VanDoorn A, Kallenbach M, Borquez AA, Baldwin IT, Bonaventure G: Rapid modification of the insect elicitor $\mathrm{N}$-linolenoyl-glutamate via a lipoxygenase-mediated mechanism on Nicotiana attenuata leaves. Bmc Plant Biol 2010, 10:164-174

45. Lee S, Hirt H, Lee Y: Phosphatidic acid activates a wound-activated MAPK in Glycine max. Plant J 2001, 26(5):479-486.

46. Halitschke R, Kessler A, Kahl J, Lorenz A, Baldwin IT: Ecophysiological comparison of direct and indirect defenses in Nicotiana attenuata. Oecologia 2000, 124(3):408-417.

47. Li L, Li CY, Lee Gl, Howe GA: Distinct roles for jasmonate synthesis and action in the systemic wound response of tomato. Proc Natl Acad SCi U S A 2002, 99(9):6416-6421.
48. Li CY, Schilmiller AL, Liu GH, Lee Gl, Jayanty S, Sageman C, Vrebalov J, Giovannoni JJ, Yagi K, Kobayashi Y, Howe GA: Role of beta-oxidation in jasmonate biosynthesis and systemic wound signaling in tomato. Plant Cell 2005, 17(3):971-986.

49. Felle HH, Zimmermann MR: Systemic signalling in barley through action potentials. Planta 2007, 226(1):203-214

50. Malone M: Rapid, long-distance signal transmission in higher plants. Adv Bot Res 1996, 22:163-228.

51. Rhodes JD, Thain JF, Wildon DC: The pathway for systemic electrical signal conduction in the wounded tomato plant. Planta 1996, 200(1):50-57.

52. Zimmermann MR, Maischak H, Mithofer A, Boland W, Felle HH: System potentials, a novel electrical long-distance apoplastic signal in plants, induced by wounding. Plant Physiol 2009, 149(3):1593-1600.

53. Maffei M, Bossi S, Spiteller D, Mithofer A, Boland W: Effects of feeding Spodoptera littoralis on lima bean leaves. I. Membrane potentials, intracellular calcium variations, oral secretions, and regurgitate components. Plant Physiol 2004, 134(4):1752-1762.

54. Mousavi SA, Chauvin A, Pascaud F, Kellenberger S, Farmer EE: GLUTAMATE RECEPTOR-LIKE genes mediate leaf-to-leaf wound signalling. Nature 2013, 500(7463):422-426.

55. Kessler A, Baldwin IT: Defensive function of herbivore-induced plant volatile emissions in nature. Science 2001, 291(5511):2141-2144.

56. Steppuhn A, Gase K, Krock B, Halitschke R, Baldwin IT: Nicotine's defensive function in nature. PLOS Biol 2004, 2(8):E217

57. Schuman MC, Barthel K, Baldwin IT: Herbivory-induced volatiles function as defenses increasing fitness of the native plant Nicotiana attenuata in nature. elife 2012, 1:e00007.

58. Fragoso V, Rothe E, Baldwin IT, Kim SG: Root jasmonic acid synthesis and perception regulate folivore-induced shoot metabolites and increase Nicotiana attenuata resistance. New Phytol 2014, 202(4):1335-1345.

59. Philippe RN, Ralph SG, Mansfield SD, Bohlmann J: Transcriptome profiles of hybrid poplar (Populus trichocarpa $\mathrm{x}$ deltoides) reveal rapid changes in undamaged, systemic sink leaves after simulated feeding by forest tent caterpillar (Malacosoma disstria). New Phytol 2010, 188(3):787-802.

60. Rostas M, Eggert K: Ontogenetic and spatio-temporal patterns of induced volatiles in Glycine max in the light of the optimal defence hypothesis. Chemoecology 2008, 18(1):29-38.

61. Krugel T, Lim M, Gase K, Halitschke R, Baldwin IT: Agrobacterium-mediated transformation of Nicotiana attenuata, a model ecological expression system. Chemoecology 2002, 12(4):177-183.

62. Roda A, Halitschke R, Steppuhn A, Baldwin IT: Individual variability in herbivore-specific elicitors from the plant's perspective. Mol Ecol 2004, 13(8):2421-2433.

63. Zhang S, Klessig DF: Salicylic acid activates a 48-kD MAP kinase in tobacco. Plant Cell 1997, 9(5):809-824.

doi:10.1186/s12870-014-0326-z

Cite this article as: Hettenhausen et al:: Fatty acid-amino acid conjugates are essential for systemic activation of salicylic acid-induced protein kinase and accumulation of jasmonic acid in Nicotiana attenuata. BMC Plant Biology 2014 14:326.

\section{Submit your next manuscript to BioMed Central and take full advantage of:}

- Convenient online submission

- Thorough peer review

- No space constraints or color figure charges

- Immediate publication on acceptance

- Inclusion in PubMed, CAS, Scopus and Google Scholar

- Research which is freely available for redistribution 